\title{
Semiótica, ideología y poder: Robert Hodge y Gunther Kress
}

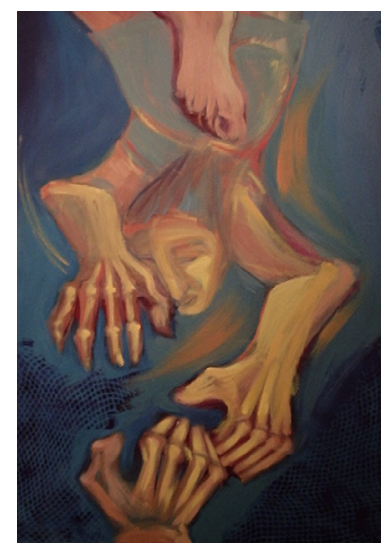

Ignacio Pérez González (entrevista, traducción y notas)

FFyL-Universidad de Buenos Aires, Argentina

ignacio.perez@gmail.com

Werner Pertot (entrevista, traducción y notas)

FFyL-Universidad de Buenos Aires, Argentina

laotrajuvenilia@hotmail.com

"Una vez fuimos siameses", bromea el lingüista Gunther Kress sobre su etapa de trabajo en colaboración con Robert Hodge. Ambos trabajaron juntos hasta comienzos de los años noventa. Fruto de esa colaboración surgió el libro El lenguaje como ideología, que comentan en esta entrevista, realizada en octubre de 2015 en el Instituto de Lingüística de la UBA, donde ambos habían asistido como plenaristas invitados al Congreso Internacional de la Aled (Asociación Latinoamericana de Estudios del Discurso).

Robert Hodge es un semiólogo australiano y uno de los fundadores de la corriente conocida como Lingüística Crítica. Ha trabajado desde diversos campos, como el estudio de medios, el marxismo, los estudios poscoloniales. Actualmente es profesor en la Universidad Western Sydney (Australia). Estudió en Cambridge y luego dio clases en la Universidad de East Anglia, donde fue su trabajo en colaboración con Kress.

Gunther Kress, por su parte, da clases de semiótica y educación en el Instituto de Educación de la Universidad de Londres. Al igual que Hodge, trabajó en estudios interdisciplinarios y multimodales, particularmente en la relación del análisis del discurso con las teorías de aprendizaje. Investigó también los cambios producidos por los medios masivos de comunicación desde la prensa escrita a la pantalla. En 2012, lo nombraron Miembro de la Orden del Imperio Británico por sus servicios como académico.

\section{¿Cómo afecta el lenguaje a la percepción humana?}

Gunther Kress: La forma tradicional de pensarlo es la de Benjamin Lee Whorf, es decir que el lenguaje te provee las categorías para pensarlo. Pienso que esto está bien, excepto porque los ambientes en los que nos encontramos son muy pequeños. Las conversaciones casuales, interacciones con alguien que conocés o que vivís de la misma forma. Esto te hace usar los recursos lingüísticos y te hace redefinirlos. Whorf plantea categorías que son muy estables, nociones estables, y yo diría que los recursos cambian 
constantemente, cambia mi idea del mundo, lo que tengo que hacer. El lenguaje (escrito o hablado) es una de las muchas formas en las que encuentro el mundo, hago ciencia en el mundo y genero mi sentido del mundo.

Robert Hodge: Quisiera enfatizar el enfoque semiótico, incluso llamarlo una "revolución semiótica" en nuestro lenguaje actual. El lenguaje disponible, escrito o hablando, es uno entre muchos. Lo que afecta la percepción es lo que ingresa vía todos estos otros lenguajes, que tienen categorías, algunas de las cuales son comunes, pero otras difieren en los distintos modos de lenguaje. Se abre otro juego. La idea de que el lenguaje verbal determina el pensamiento se vuelve un sinsentido. Alrededor del lenguaje verbal hay otros sistemas científicos que deben ser percibidos para tener algún efecto. No se puede decir que el lenguaje determina la percepción si la percepción es el canal desde el cual viene el lenguaje, del que vienen todos los lenguajes. Esto nos lleva a pensar lo que es el pensamiento. El pensamiento consiste en estructuras profundas y procesos, que emergen a lo largo de un tiempo extenso, afectados por el grupo social en el que se vive, y que integran algunos de los mensajes en la multiplicidad de canales. El foco debe estar puesto en que estos pensamientos profundos sólo pueden ser analizados a través del lenguaje, así que -de repente- el lenguaje tiene un rol clave. Pero no porque el lenguaje determine el pensamiento, sino porque el lenguaje está determinado por el pensamiento.

GK: En este punto debo diferir fundamentalmente en querer deshacernos de la noción del lenguaje.

RH: Perdón, ¿querés deshacerte de la noción del lenguaje?

GK: $\mathrm{Si}$, eso quiero.

RH: Es lo que estoy diciendo yo.

GK: No, lo que estás diciendo es que, al final, solo se pueden entender las categorías del lenguaje.

RH: Me refiero a las categorías profundas de todos los lenguajes.

GK: O se puede decir que las categorías abstractas semióticas se materializan en distintos recursos semióticos.

RH: Sí, eso es lo que buscaba decir.

GK: Para mi hay una distinción entre lo fisiológico, el sensorium que tenemos, y nosotros -no vos y yo, sino un nosotros colectivo- tenemos un entendimiento real del sensorium, lo que está muy definido convencionalmente. En el sensorium está el olfato y hay que pensar hasta qué punto lo semiótico y lo fisiológico interactúan. Debemos pensar en ello. Incluso si tenemos muchas formas de representar semióticamente el mundo y de interactuar con él, tenemos limitaciones en función de lo que es capaz el sensorium de hacer. Mi razón de ir alejándome de la lingüística es que aplica un filtro brutal a cómo pensamos el mundo, desde mi punto de vista. 
GK: Yo usaría una metáfora muy simple: la posición. Siempre veo el mundo desde una posición. Por ejemplo, estamos en este cuarto acá, juntos. Cuando nos vayamos, alguien podría decirnos: “¿cómo era el cuarto?”. Uno podría relatar: "Bueno, en realidad, lo principal era la puerta", y vos podrías decir: "Bueno, lo principal era la ventana ${ }^{1}$. Y de hecho compartimos la mesa, pero en realidad esa misma mesa también se ve distinta con aquello como fondo. ¿Se entiende a qué me refiero? La noción de posicionamiento -y pensar en qué tipos de posiciones hay en un montón de formas distintas- no puede evitarse. Es lo que llamamos posición o perspectiva o, desde un punto de vista más amplio, como una cuestión sistemática, la ideología. Y entonces, qué noción de ideología te interesa, la de noción de Marx o una menos específica. Creo que no podemos hablar o representar o involucrarnos con una palabra si no es con una posición específica en un momento específico.

RH: Por un lado, estoy de acuerdo con el modo en que Gunther toma la ideología, pero también puedo interpretarlo en un sentido mucho más estrecho -porque la ideología es un concepto con el que es muy fácil casarse- y le daría un significado mucho más fuertemente social. Y así podría convertir tu pregunta en algo más parecido a "¿Cómo un conjunto de encuadres del mundo, dados por un posicionamiento social, acciona sobre las elecciones que tomamos?". Y mi respuesta sería que es algo que tiene que ser considerado como intermitente. Darle un rol tan crucial a la ideología... es decir, es importante, pero es intermitente. La vida cotidiana es llevada a cabo de acuerdo a cosas que podrían ser llamadas ideologías, pero ciertamente no en el sentido fuerte en el que nos referimos a ideología.

GK: No me refiero a eso. Pensando en una escala más pequeña, quiero ver continuidades, retomando tu noción como fractales. La posición acá en el cuarto de la que hablaba, era en un sentido totalmente banal, tiene un efecto. Pero hablo de mi posicionamiento social, en distintos entornos, en distintos contextos sociales, contextos institucionales, etcétera. Quiero llevar esa noción más allá. Y en cierto punto voy a llamar a esto "ideología". Excepto, quizás, que creo que lo que tomo de tu charla de hoy es que tengo que repensar eso. Todavía estoy muy centrado en cosas a nivel micro, que cambian de un momento a otro, aunque tienen estabilidad, desde la estabilidad de la persona que en cierto punto se encuentra con el mundo, y esa estabilidad viene, por supuesto, de las comunidades. Pero cuando pienso en la actual Inglaterra, todas estas cosas están fragmentadas o están por serlo. Y para mí, la pregunta sería "cuál es la diferencia entre una posición ético-moral y una ideológica". Y la gran pregunta de hasta qué punto puede esto hacer referencia a la clase y no a otras categorías sociales.

\section{¿Cómo estudiaron la ideología en el lenguaje?}

GK: Voy a dejar hablar a Bob (risas)

RH: Bueno... están los diferentes sentidos de "ideología" que estamos usando. Y yo creo que ahí yace el problema con la ideología. Es un término que está en circulación, que tiene un amplio rango de significados. Y podemos tratar de fijarla en uno u otro sentido, pero la palabra sigue recuperando ese rango de significados. Pero en esta discusión, una cuestión para ver es que "ideología" tiene un sentido más estrecho, más políticamente modificado. Lo que quiero decir es que cuanto más expandido está su sentido, tanto más difícil es utilizar el término para explicar alguna cosa. Ideología, en un sentido más estrictamente político, me gusta verlo como organizado por
1. El entrevistado enfrentaba a la puerta y el entrevistador veía la ventana. 
múltiples determinaciones. En cualquier acto de habla -o en cualquier acto, incluyendo su representación en discurso- hay una dimensión ideológica que es una estructura más genérica derivada. Está vigilada por grupos. Creo que es útil reconocer que el pensamiento es vigilado, vigilado socialmente. Lo social no es sólo lo que nos es inculcado de jóvenes. El lenguaje y el pensamiento son sociales. Pero, en esta discusión, creo que hay una diferencia entre ese proceso, que es en todo permeable, y una suerte de determinación ideológica.

Pero creo que, para ilustrar lo que digo, voy a usar la ideología del género. Veo eso como una ideología, que se refuerza, se pueden mostrar los procesos con los que es inculcada y me lleva a clasificar sin esfuerzo lo que entendemos aquí en términos de género. Si hay una mujer acá, esa ideología se mete en el medio. Y mi entendimiento de cómo ese tipo de fuerza social funciona es que aparece casi completamente formada e interrumpe el flujo de interacción social. Y eso impregna todo y de pronto estoy tratándote como masculino y a esta mujer imaginaria como femenina. Y todos están sujetos a esta misma ideología y por lo tanto todos nos posicionamos en torno a esto. Y quiero decir que hay otras fuerzas sociales influyendo en cómo interactúo con vos y esta mujer imaginaria de formas distintas. Incluso socialmente, no independientemente de las determinaciones ideológicas, creo que la ideología en ese sentido acotado, es una fuerza formidable que es muy necesario examinar y disputar, que es productivo utilizar el término "ideología" para eso. Pero como digo, podrías usar otra palabra para ideología, no necesita tener ese sentido. Sólo la uso con ese significado para esta conversación.

En su caso, empezó en el análisis del discurso y luego tomó un enfoque más semiótico...

RH: Claro, escribimos eso juntos.

GK: Cuando escribimos Lenguaje como ideología en el sentido que veníamos diciendo.

\section{Sería el sentido marxista de "ideología".}

GK: Sí. Solo que dejamos sin debatir...

RH: Yo diría que fuimos criticados por no utilizarla en el sentido marxista completo.

GK: Sí. Por eso es que quiero decididamente señalar la continuidad si me estoy moviendo hacia un dominio donde el poder y la política como poder se convierten en la cuestión. Cuando trabajamos juntos con la ideología, de hecho, empezamos con una suerte de semiótica más cercana -en los términos del marco de Halliday- al tipo de cláusulas, o bien -al mismo tiempo, en ese momento- a la gramática de casos de Chomsky. Empezamos con la forma en la que percibimos el mundo fisiológicamente o físicamente y procedimos de esa percepción hacia el modo en que el mundo es lingüísticamente nombrado. Pero, cuando terminamos el libro, pensamos que realmente lo que queríamos describir es el caso, en una forma distinta. Estas categorías, si las abstraes un poco, funcionan en otros dominios. Dijimos: Bob ya se estaba volviendo de Inglaterra a Australia y teníamos este largo camino donde pensábamos investigar en otras áreas, porque mirarlo solo desde el sistema lingüístico no es suficiente. Pero se volvió muy difícil porque yo estaba en Inglaterra, él estaba en Australia. 
Diferentes lugares. Pero intentamos trasladar las categorías de Lenguaje como ideología más o menos a este dominio más amplio. ${ }^{2}$ Otra cuestión, que sale de lo que dijiste esta mañana ${ }^{3}$, es que empezamos a trabajar con el signo lingüístico, y en la diferencia entre significante y significado. Y en que esa relación entre significante y significado no es arbitraria sino motivada o icónica. Y esto plantea una gran diferencia en, por ejemplo, las palabras como "ideología" o como "signo" plantean con un significante distintos significados según los distintos ambientes en los que los usas con distintos propósitos.

\section{¿Cómo nos puede ayudar la Lingüística Crítica o el Análisis Crítico} del Discurso (ACD) a entender el lugar que ocupan los medios?

GK: Necesitaría más o menos dos días, como mínimo, para contestar esa pregunta (Risas). Lo que creo es que la Lingüística Crítica y el ACD no pudieron enfocarse en cómo esto que vagamente podemos llamar mensaje fue construido para lograr determinados propósitos. Esto es lo que la ideología intenta hacer. No planteamos realmente una teoría de la comunicación -aunque puede que no estés de acuerdo con lo que digo (refiriéndose a RH)-, creo yo. No dijimos cómo las personas que reciben esto... Bueno, en realidad, sí lo hicimos, porque en el libro Social Semiotics vos hiciste una suerte de comentario del hecho de que los medios, en un punto de la historia de Inglaterra cuando se elegía un nuevo líder del partido laborista, en última instancia intentaron descartar un líder posible y las personas pudieron tomar una decisión completamente errada. Acá aparece un sentido fuerte de la ideología, como puesta encima, como que el mensaje se mantiene, en un sentido kafkiano. Lo leyeron en otros países y lo tradujeron, lo transformaron. Pero no era una teoría completa de la comunicación, que es lo que necesitamos ahora, donde una persona crea un objeto, un objeto semiótico, y una persona que lo recibe, lo rehace en función de los recursos con los que cuenta. Y por supuesto está localizado socialmente: con los recursos que tengo en mi historia social y mi posición social, y se reintegra en una noción social. Volviendo a tu pregunta, así fue cómo llegamos a la semiótica, porque pensamos que nuestro proyecto no estaba completo si lo enfocábamos sólo en el lenguaje.

RH: Usando las palabras de mi ponencia (la cuestión del PatoNejo) ${ }^{4}$, aunque incluimos a los medios no los teníamos claramente en vista... Necesitábamos centrarnos en los procesos textuales, necesitábamos hacer esto para ver cuán fundamental es no naturalizar o dar por supuestos los procesos textuales y las interpretaciones involucradas. Eso incluye teorías psicológicas y sociales sobre los medios, algunas de las cuales llegaron a ser muy simplistas y poco realistas. Pero, realmente e indudablemente, pudimos ser malinterpretados: son procesos complejos, no se los puede naturalizar, deben estudiarse. Con el libro Social Semiotics estaba pensando cosas similares a las de Gunther en relación a la televisión. En un sentido, lo que hicimos con estas diferentes posiciones en la problemática de la comunicación fue atender a distintos puntos y llegar a similares conclusiones. Cuando comenzamos, ni siquiera sabía que no tenía nada para decir sobre estas cosas porque era solo una teoría de producción, no estaba interesado en la recepción.

Eliseo Verón habla de la gramática de la producción y de la gramática de la recepción para diferenciar estos puntos. Pero, además, está el hecho de que los medios le pueden hablar a una gran cantidad de personas...
2. Hodge, B. y G. Kress (1988) Social semiotics. Cambridge. Polity Press 3. Hodge, R. (2015) “Discursive constructions of Latin America/ America Latina: A multiscalar analysis", pronunciada en el marco del XI Congreso Internacional de la ALED, Ciudad Autónoma de Buenos Aires. Recuperado de: https://www. youtube.com/watch?v=ndOG8hsv_b8
4. Hodge, R. (2015) “Discursive constructions of Latin America/ America Latina: A multiscalar analysis", pronunciada en el marco del XI Congreso Internacional de la ALED, Ciudad Autónoma de Buenos Aires. Recuperado de: https://www. youtube.com/watch?v=ndOG8hsv_b8 
GK: Los medios establecen la agenda. Desde la perspectiva de una teoría de la comunicación y del cambio, decimos que ponen a disposición una serie de preguntas o disparadores. E incluso aunque cada lector de ese medio (usando una noción de Stuart Hall) pueda rechazar y decir "ni siquiera voy a mirar esto" o puede leerlo desde su posición y transformarlo desde esa posición, ese "esto" o esa transformación se hacen sobre algo que surge de una propuesta o disparador que alguien ya construyó. Eso es lo que hacen los medios. En Inglaterra, por ejemplo, hay cosas que simplemente no aparecen (imagino que es igual en Argentina). Directamente no aparecen en los medios. Y por lo tanto, no pueden rebatirse o responderse. Desaparecen de la visión a menos que uno tenga la capacidad para darse cuenta y decir "no me están diciendo lo suficiente, quiero saber más". Así que sí, creo que es así como los medios establecen la agenda. E incluso cuando no pueden garantizar la interpretación o transformación de lo que ponen en la agenda, saben que eso es lo que queda.

Hay una particularidad de la realidad argentina. Hasta 2015, hubo un conflicto acá entre el Gobierno y uno de los principales grupos mediáticos locales, el Grupo Clarín. Esto produjo una polarización en los medios, que son muy enfáticos en la forma en que construyen los títulos, los textos. Son completamente a favor o completamente en contra. Y está todo definido en términos de nosotros o ellos. En este tipo de confrontación política, ¿qué rol consideran que tienen los signos o el lenguaje?

GK: En Inglaterra no hay medios anti gobierno. Pero nosotros estábamos en la Universidad de East Anglia, cuando escribimos juntos el libro Language and control. En ese libro escribió uno de nuestros colegas, Tony Trew, de Sudáfrica, que daba un curso de filosofía y lenguaje. Y tomamos algunos de sus materiales de los conflictos de ese entonces en Sudáfrica, Rodhesia ${ }^{5}$ y Mozambique. Y pudimos comparar, cuando tuvimos acceso a los diarios, las representaciones de un evento específico en distintos diarios: un diario controlado por el gobierno sudafricano o uno de Tanzania. En el caso de Argentina tienen esta situación en un sólo país (esto era en países diferentes, porque en Sudáfrica no se podía tener un medio anti gobierno, como no se puede tener en Inglaterra en este momento). Lo que tenés es una representación del mundo: "15 terroristas murieron en una manifestación" o "asesino colonialista mató a 15 pacíficos civiles".

\section{Dos realidades diferentes.}

GK: Dos diferentes, sí. Y si uno tiene una teoría que dice que el signo está formado según el interés el hacedor del signo (ésa sería mi teoría actual), entonces diría que entendemos algo de cómo funcionan los medios en Argentina y quién controla los medios. Podemos decir (en Inglaterra Murdock controla el 75\% de los medios impresos) "la posición y el interés de este hacedor de signos particular es éste y puedo predecir cómo va a ser ese signo". Y podés tener una teoría que dice que el interés del hacedor del signo está siempre presente en el signo y que el que se involucra con el signo pone su interés en la reproducción o resignificación de ese signo. Podría ser útil desde la secundaria tener unos simples ejercicios proponiendo "pretendé que sos tal actor y presentá este evento desde su postura". O sea... no querés generar una población esquizofrénica (risas)

Volviendo a la polarización y los medios: en la recepción de estos medios, se forman ciertas comunidades. Los receptores tienen una reacción muy poderosa a estos signos.
5. Actual Zimbabue, Estado ubicado al sur de África. El trabajo de T. Trew al que hace referencia Kress es "Teoría e ideología en acción" (en Lenguaje y control. México. FCE pág. 127-158). 
GK: Son comunidades que se caracterizan por el periódico que compran. Es el mundo que quieren conocer. Supongo que en algún punto funciona como con los clubes de fútbol. Se vuelven comunidades irreconciliables. Y la forma que utilizan para comunicarse deben ser, mayormente, formas violentas, imagino.

RH: Respecto de este problema -un fenómeno en el que hace mucho que estoy interesado-, es cómo los medios pueden producir, no solo ciertos sesgos por deslizarse hacia la derecha en las representaciones de eventos, sino también otros efectos semióticos: una cierta esquizofrenia, por ejemplo. Si después de un tiempo la gente no puede entender o no sabe dónde está la verdad, reacciona de formas extrañas. En Australia, por ejemplo, hay un sesgo obvio y sistemático en contra del concepto de cambio climático de parte de los medios de Murdock. A pesar de que la población tiene acceso a otras miradas, hay una presentación consistente de un tipo particular de visión al respecto. Pero una consecuencia de la preponderancia de este tipo de mensaje -como el de los medios de Murdock- es una cierta ya no creencia en que el cambio climático es falso, sino un cierto pesimismo, un sentimiento de que no sabemos (cuando en realidad probablemente sí sabemos).

GK: Uno de los efectos es llamado en Inglaterra "la disolución del votante firme".

RH: Exacto. Sí.

GK: Se trata de personas que simplemente se rehúsan a formar parte del proceso. El actual gobierno de Inglaterra entonces es electo por el 37\% de las personas que votan. Es un número muy pequeño de personas el que le dio a este Gobierno la mayoría absoluta. Pero la mayoría en verdad, se rehúsa a participar en el proceso electoral.

RH: Veo que es un efecto que no tomamos en cuenta. La consistencia de las mentiras que la gente puede creer, sobre el cambio climático. Y luego hay un efecto posterior en el estado semiótico de la mente. Las mentiras generan o producen una falta de confianza, falta de compromiso. En los países en los que la prensa está sistemáticamente dominada por los Gobiernos la gente no cree en la prensa, escucha su versión dominante, pero tienen esta especie de disolución, falta de compromiso, dado que no pueden saber cuál es la verdad.

GK: En Inglaterra, en parte los periódicos están en crisis porque la gente ya no los compra. La circulación cayó masivamente. Muchos pueden leer online. No sé, en verdad, qué hacen en lugar de leerlos. Antes de la invasión a Irak, solía leer The Guardian. Ese diario pasó a estar a favor de la invasión y comencé a negarme a comprarlo, porque se convirtió en parte de la centroderecha mainstream. Pero pasé de The Guardian a leer Daily Telegram, que es una posibilidad para la clase media: lee a The Guardian o al Telegram. Hay un efecto de clase o de educación. El Telegram se dirige a personas de derecha, pero lo hace de una manera distinta que el Daily Mail, que es brutal y burdo.

RH: Volviendo a lo que planteaba como un sentido básico, como el problema de un código mezclado, es la idea de una corrupción semiótica. La idea de que la corrupción plantea efectos individuales, pero también lleva a una ruptura en una forma fundamental de hacer sentido, que es una patología. 
Esto ocurre en México. Todos saben que el gobierno miente. Puedo sacar conclusiones obvias cuando estoy afuera. Desde afuera parece muy obvio. Se presentan dos partidos igualmente corruptos en forma profunda, uno ligeramente a la izquierda. Y se plantea que una de las pocas distinciones que sobreviven todavía en este colapso de categorías semióticas es la diferencia entre el PRI y el PAN. Piensan que solo pueden decidir entre lo que ahora se ha convertido en una sola entidad. El efecto de lo que sucedió es muy sutil. Estos procesos son tan sutiles y toma mucho tiempo poder identificarlos. La única forma de hacerlo es tomar largas franjas de tiempos (por ejemplo, 20 años hacia atrás) y hacer una investigación más larga. Estos colapsos semióticos tienden a ocurrir en un tiempo corto, donde A pasa a ser B, B pasa a ser Y. Es realmente una situación PatoNejo ${ }^{6}$, como dije en mi ponencia. Las personas deben enfrentarse a esta situación de partidos de izquierda-derecha, que todo el tiempo están mutando. Y ya no pueden distinguir entre la derecha y la izquierda.

GK: Ustedes dos hacen sociolingüística así que saben sobre las conversaciones con latiguillos. En Inglaterra no hay dos conversaciones sin que cada enunciado sea seguido de un "Para serte honesto". Esto no ocurría antes. ¿Qué ha pasado para que todo el mundo diga "Para serte honesto"? Lo que ocurre para mi es la corrupción terrible de en qué puede confiarse, entonces aparece esta rutina de "para serte honesto". Te estoy diciendo un montón de mentiras y "para serte honesto". Los latiguillos, en verdad, son un tipo de signo muy interesante. Un síntoma...

\section{Que uno tiende a no prestarle atención.}

GK: Pero son signos de... por qué diablos estás diciendo “¿Sabés lo que quiero decir?".

RH: No sabés qué quiero decir.

GK: No lo sabés.

RH: Y no quiero decir nada. (Risas)

GK: Por eso son signos muy interesantes.

\section{Ustedes mencionaron a Whorf. ¿Se considerarían relativistas} lingüísticos?

GK: Yo diría que construimos nuestra mirada del mundo desde la posición social en la que estamos, no individualmente, sino con otros. Lo que se conoce como constructivismo es lo que yo creo que, más o menos, es lo que podemos hacer. En Lenguaje como ideología hablamos de la relación entre lenguaje y poder, poder y lenguaje. Pero no es una cuestión totalizadora. Si me llevan a un hospital para operarme, me gustaría pensar que de diez cirujanos ninguno es tan constructivista.

RH: Todos hablan en inglés, dirías vos. (risas)

GK: En Inglaterra, todos hablan inglés. Pero entiendo que la idea del dolor no es construida. Y que los que me atienden tienen alguna idea de la medicina. Y Halliday era whorfiano. Las categorías del lenguaje que tenemos construyen nuestra visión de mundo. Esto se completa con la noción marxista de que las categorías que construimos, las construimos en las condiciones sociales en las que estamos. Así que una se vincula a la otra. Y
6. Hodge, R. (2015) “Discursive constructions of Latin America/ America Latina: A multiscalar analysis", pronunciada en el marco del XI Congreso Internacional de la ALED, Ciudad Autónoma de Buenos Aires. Recuperado de: https://www youtube.com/watch? $v=$ ndOG8hsv_b8 
por supuesto una vez que tenés esta categoría definen tu visión de mundo. Luego replantean tus condiciones sociales. Es dialéctica y cambio.

RH: Para clarificar mi visión sobre Whorf, lo que discuto es que la idea del lenguaje es tan solo el lenguaje verbal. Creo que si lo cambiás al rango total de la semiótica, entonces sí. El relativismo es interesante. Whorf no era relativista absoluto, sino relativista en una forma multiescalar. Entonces, el inglés y el español son más o menos lo mismo si lo comparás con las lenguas indígenas. La idea de que todos los lenguajes son igualmente inconvertibles no es lo que él planteaba. Los lenguajes europeos de dominación tienen una gran cantidad de ocultamiento de los hechos de los que son responsables de la masiva dominación de las Américas. Esto incluye a los españoles, portugueses e ingleses. Tienen más en común como lenguajes de dominadores que los lenguajes de resistencias. Entonces, las categorías sociales son mucho más relativistas que la idea de que cada lengua es siempre igualmente y completamente autónoma. Lo que hay que pensar es que donde haya voluntad política, las personas dominadas pueden estar atravesadas por el lenguaje del conquistador. Ellos pueden entender el lenguaje de los conquistadores. Entonces, el principio relativista puede ser usado para cerrarle la puerta a observar la dominación colonial, lo que es políticamente falso. Las personas indígenas en Australia pueden con gran facilidad ver el lenguaje de los ingleses. Las personas indígenas en América pueden ver, también. Así que hay un peligro en el uso de las categorías de Whorf y el marco whorfiano. Él, en verdad, estaba oponiéndose a la dominación de los ingleses y los españoles en las Américas. Él no lo decía así, pero ese era su proyecto político: que los colonizadores de las Américas eran estúpidos e ignorantes en relación a los colonizados. Él tuvo que ser indirecto y por ser tan indirecto no fue entendido bien. Así que ese era Whorf.

Muchas gracias.

GK: Si nos dan más bizcochos, pueden hacernos más preguntas.(Risas) 